\title{
Insécurité Alimentaire des Ménages Agricoles et Stratégies de Résilience au Sahel : Cas de la Vallée de Goulbi Maradi, Niger
}

\author{
Nouhou Dan Ango Harouna, Doctorant \\ École Doctorale des Lettres, Arts et Sciences de l'homme et de la Société, \\ Université Abdou Moumouni de Niamey, Niger \\ Sadda Abou-Soufianou, Doctorant \\ Université Dan Dicko Dankoulodo de Maradi, ADS Maradi, Niger \& \\ UMR 242-iEES-Paris, IRD France -Nord, Bondy cedex, France \\ Yamba Boubacar, Enseignant Chercheur
}

Département de Géographie, Faculté de Lettres et Sciences Humaines,

Université Abdou Moumouni, Niger

Doi: 10.19044/esj.2019.v15n18p96 URL:http://dx.doi.org/10.19044/esj.2019.v15n18p96

\section{Résumé}

La présente étude met en évidence la problématique de l'insécurité alimentaire ainsi que les stratégies adoptées par les ménages agricoles pour une résilience face aux chocs. Elle a été conçue à travers les recherches documentaires, les visites du terrain ainsi que les enquêtes socio-économiques. En outre, cette étude nous a permis d'appréhender les déterminants de l'insécurité alimentaire des ménages de la commune de Tibiri. Ces déterminants ne sont autres que les facteurs naturels et humains. Tout de même, le niveau de la couverture alimentaire et surtout les stratégies de résiliences pour rebondir aux chocs ont été déterminées. De ce fait, les résultats révèlent que $48 \%$ des ménages souffrent d'une insécurité alimentaire sévère ; $43 \%$ souffrent d'une insécurité alimentaire modérée tandis que 7\% sont à risque et seulement $2 \%$ sont en sécurité alimentaire. Au regard de tout ce qui précède, on peut dire que face à cette situation, plusieurs stratégies de résiliences apparaissent. Elles sont nées du savoir traditionnel des paysans et permettent d'accroitre la production pour mieux renaitre suite aux dommages causés par les phénomènes naturels du milieu.

Mots-clés: Insécurité alimentaire, Résilience, savoir traditionnel, Niger 


\title{
Food Insecurity of Agricultural Households and Resilience Strategies in the Sahel: Case of Goulbi Maradi Valley, Niger
}

\author{
Nouhou Dan Ango Harouna, Doctorant \\ École Doctorale des Lettres, Arts et Sciences de l'homme et de la Société, \\ Université Abdou Moumouni de Niamey, Niger \\ Sadda Abou-Soufianou, Doctorant \\ Université Dan Dicko Dankoulodo de Maradi, ADS Maradi, Niger \& \\ UMR 242-iEES-Paris, IRD France -Nord, Bondy cedex, France \\ Yamba Boubacar, Enseignant Chercheur
}

Département de Géographie, Faculté de Lettres et Sciences Humaines,

Université Abdou Moumouni, Niger

\begin{abstract}
This paper focuses on highlighting the problem of food insecurity as well as the strategies adopted by farm households for resilience to shocks. It was carried out through documentary research, field visits, and socioeconomic surveys. In addition, this study allowed us to understand the determinants of household food insecurity in the municipality of Tibiri. These determinants are none other than natural and human factors. All the same, the level of the food coverage and especially the resilience strategies to bounce back to shocks has been determined. The results showed that $48 \%$ of households suffer from severe food insecurity, $43 \%$ are moderately food insecure, $7 \%$ are at risk, and only $2 \%$ are food secure. In view of all the above, it can be stated that in the face of this situation, several resilience strategies appear. They are born of the traditional knowledge of the peasants and allows a significant increase in the production to better rebirth the damages caused by the natural phenomena of the medium.
\end{abstract}

Keywords: Food insecurity, Resilience, Traditional knowledge, Niger

\section{Introduction}

La sécurité alimentaire des ménages et la lutte contre la pauvreté constituent de nos jours une des préoccupations majeures dans plusieurs pays du monde (Mortimore \& Adams, 2001; Ouédraogo, Kaboré, \& Kienou, 2007). En Afrique subsaharienne, l'agriculture est un secteur clé de l'économie et son 
intensification et diversification restent une priorité (Eromosele, Arogundadea, Eromosele, \& Ademuyiwa, 2008).

Cependant, le Niger connaît depuis plusieurs décennies, de fortes variabilités climatiques caractérisées par une fluctuation de la période et de la durée des précipitations, une variation de la pluviométrie annuelle, un climat de plus en plus chaud, des cycles de sécheresses, des inondations subites et imprévisibles, la dégradation des sols, des vents violents et la prolifération des maladies et ravageurs (Yabi \& Afouda, 2012).

Ce changement climatique, source d'inquiétude pour les agriculteurs affecte négativement les rendements agricoles par leurs impacts sur la croissance, le développement des plantes et la diversité variétale (Rahman, Luo, Hafeez, \& Sun, 2015).

La baisse des rendements agricoles en raison de mauvaises conditions pédoclimatiques conduit à coup sûr à l'insécurité alimentaire croissante, à la vulnérabilité des communautés agricoles, à la réduction des revenus des ménages et à une augmentation de la pauvreté (Deressa, Hassan, \& Ringler, 2011; Srivastava, Shakya, \& Kumar, 2012).

C'est ainsi que la recherche des solutions aux risques engendrés par l'insécurité alimentaire s'impose avec beaucoup plus d'acuité.

C'est pourquoi la lutte contre l'insécurité alimentaire est définie dans un cadre plurisectoriel tenant en compte des interactions homme-environnement (Vonthron, Dury, Fallot, Alpha, \& Bousquet, 2016).

La population tente de répondre aux changements climatiques (Vodounou \& Onibon Doubogan, 2016) prenant en compte les dimensions tant économiques que politiques, sociales, culturelles et naturelles (Heijmans, 2013).

L'objectif de cette recherche est de déterminer le niveau de l'insécurité alimentaire des ménages de la vallée de Goulbi Maradi ainsi que les stratégies adoptées pour une meilleure résilience face aux différents chocs.

Pour atteindre cet objectif, nous formulons l'hypothèse selon laquelle les impacts négatifs du changement climatique couplés avec la pression démographique sur les ressources naturelles se traduisent par la baisse de la production agricole.

Pour vérifier cette hypothèse, deux questions sont posées : (i) quelles sont les conséquences directes du croît démographique et du changement climatique sur la production? (ii) quelles sont les stratégies de résilience mises en place par la population face à ce choc tant structurel que conjoncturel ? Cette question renvoie à la problématique de la capacité de la mise en place des stratégies de résilience par les ménages. 


\section{Matériel et Méthodes}

\section{Site de l'étude}

L'étude a été menée dans la commune de Tibiri (région de Maradi), située au centre-sud de la république du Niger entre $13^{\circ} 10^{\prime}$ et $13^{\circ} 40^{\prime}$ de latitude Nord et, $6^{\circ} 30^{\prime}$ et $7^{\circ} 03^{\prime}$ de longitude Est (Figure 1), et couvrant une superficie d'environ $1200 \mathrm{~km}^{2}$.

Le climat est de type sahélo-soudanien avec deux saisons contrastées : une longue saison sèche d'octobre à mai (soit 8 mois) et une courte saison humide correspondant à la saison hivernale de juin à septembre (soit 4 mois). Il est caractérisé par une pluviométrie moyenne annuelle calculée sur 30 ans de $477 \mathrm{~mm}$, et une température moyenne annuelle relativement élevée $\left(28^{\circ} \mathrm{C}\right)$.

En termes de ressources en eau, la zone d'étude dispose d'un cours d'eau semi permanent, le "Goulbin Maradi», qui arrose sa partie Est sur environ $15 \mathrm{Km}$; et quelques mares temporaires.

La population de la commune urbaine de Tibiri est estimée à 124849 habitants en 2013, elle est essentiellement composée d'agriculteurs et d'éleveurs (Sadda, Lawali, Diouf, Illiassou, \& Mahamane, 2016).

La vegetation de la commune urbaine de Tibiri est une steppe arbustive à arborée de type sahélienne(Sadda, Lawali, Diouf, Illiassou, \& Mahamane, 2016). Elle est composée de deux strates:

$\checkmark \quad$ Une strate ligneuse constituée principalement des espèces suivantes : Acacia albida ou Gao, Balanites aegyptiaca, Prosophis africana et des Combretacées (Combretum glutinosum);

$\checkmark \quad$ Une strate herbeuse dominée par des espèces annuelles notamment des graminées du genre (Eragrostice tremula et Cenchrus biflorus). 


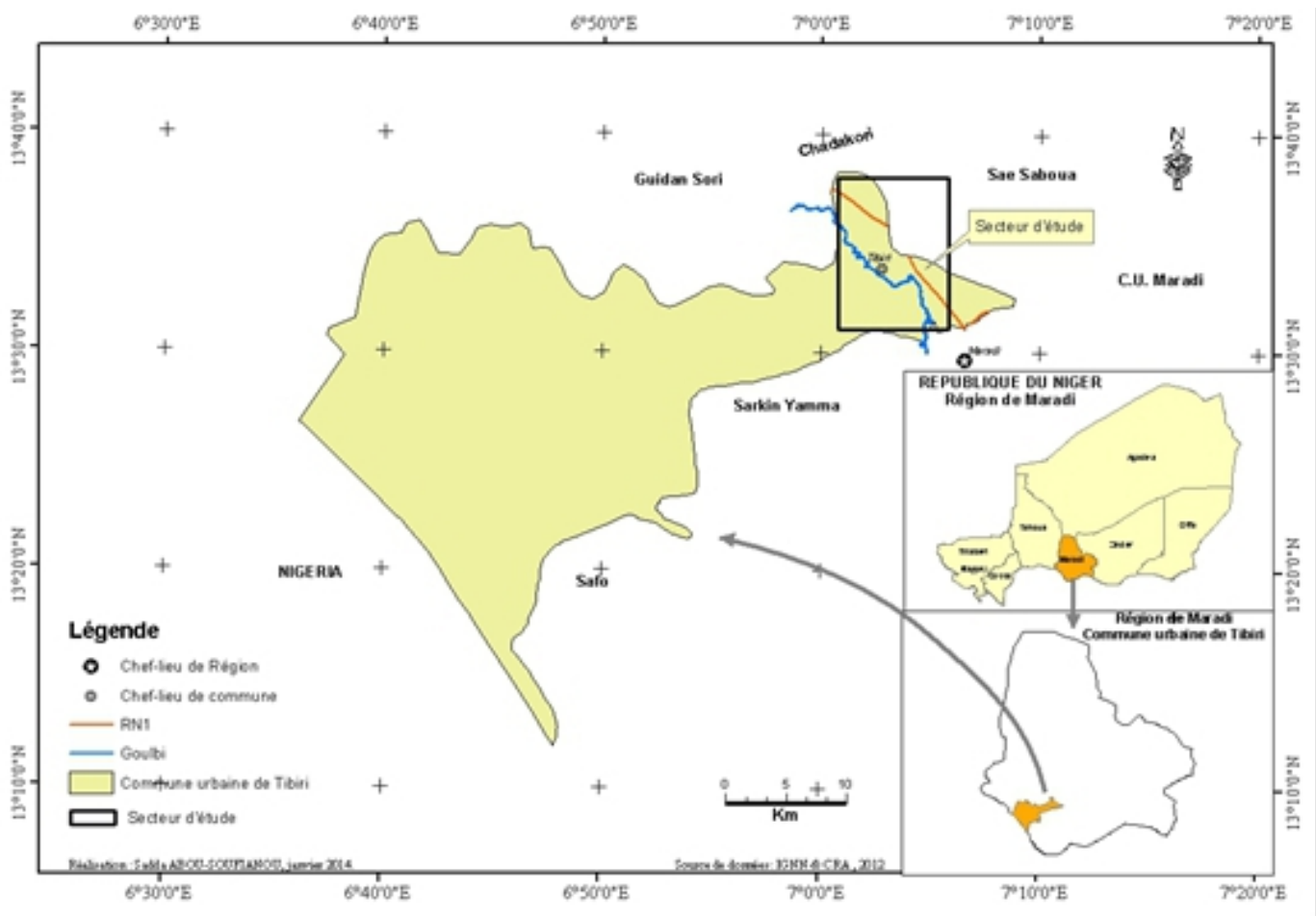

Figure 1: Présentation de la zone d'étude

\section{Approches Méthodologiques}

Une série d'enquêtes de terrain et entretiens directs ont été menés auprès des ménages agricoles, des élus locaux, des services techniques et des autorités coutumières.

Des données quantitatives et qualitatives relatives aux questions foncières, aux pratiques culturales, à la manifestation et aux ressentiments de la variabilité et changement climatique ainsi que des stratégies d'adaptation ont été collectées selon un échantillonnage stratifié aléatoire.

Pour déterminer l'échantillon, nous avons pu disposer des données démographiques actualisées du service de l'état civil de la commune de Tibiri des différents villages de ladite commune.

En effet, pour connaître les villages pouvant être retenus comme sites d'enquête, nous avons procédé à une stratification en catégories des villages longeant de part et d'autre la vallée de Goulbi Maradi; notamment les petits villages (dont la population est inférieure à 2000 habitants) et les gros villages (dont la population est supérieure à 2000 habitants).

Sur cette base, nous avons tiré au hasard au total 10 villages; soit 7 et 3 villages respectivement dans le premier et deuxième groupe des villages comme sites d'enquête. 
En outre, dans chacun des villages choisis un certain nombre de ménages à enquêter ont été échantillonnés. C'est ainsi que 10 à $11 \%$ des ménages par village dans la catégorie de moins de 2000 habitants et 12 à $13 \%$ dans celle de plus de 2000 habitants ont été enquêtés (Tableau 1).

Une série de focus groupe (avec 5 à 10 personnes) dans le but d'avoir une idée sur le système d'exploitation agricole et surtout sur les facteurs qui handicapent son bon fonctionnement a été effectué.

En plus, à l'aide d'un guide, des entretiens ont été effectués auprès des autorités administratives et coutumières; les responsables des services techniques communaux de l'agriculture, de l'hydraulique et de l'environnement.

Des analyses qualitative et quantitative ont été effectuées avec le Logiciel SPSS 19.

Tableau 1: Echantillonnage

\begin{tabular}{lllll}
\hline Villages & Population & Nombre de ménages & Echantillons & Pourcentage \\
\hline Soura Jinjuna Gata & 4000 & 294 & 36 & 12 \\
Soura Serkin Galma & 4000 & 239 & 32 & 13 \\
Soura Saraki & 1945 & 243 & 33 & 13 \\
Soura Magagin Rogo & 1200 & 140 & 16 & 11 \\
Garin Daouré & 1795 & 190 & 21 & 11 \\
Kaoukaye & 1047 & 161 & 18 & 11 \\
Tibiri ville & 30352 & 1509 & 239 & 15 \\
Waraou & 1496 & 180 & 19 & 10 \\
Mamaoua & 1197 & 48 & 6 & 12 \\
Fagagaou & 1297 & 118 & 13 & 11 \\
\hline Total & 48329 & 3253 & 433 & 13 \\
\hline
\end{tabular}

Source : Mairie de la commune urbaine de Tibiri (Octobre 2013)

\section{Résultats}

\section{Caractérisation des Ménages Enquêtés}

Les chefs de ménages enquêtés se caractérisent selon leur situation matrimoniale, leur niveau d'instruction et leur sexe. Ainsi, 190 chefs de ménages sont des polygames (soit 43,67\%) et 198 chefs de ménages sont des mariés monogames (soit 45,72\%) (Tableau 2). Trente sept (37) personnes sont des chefs de ménages en état de veuvage dont 27 femmes et 10 hommes. Seulement 8 personnes enquêtés sont des chefs de ménages célibataires. En outre, les ménages dirigés par les personnes en état de veuvage ne représentent que $8,5 \%$. Les 8 célibataires (soit 1,84\% des enquêtés) sont des jeunes garçons ayant perdu leurs parents et qui prennent la responsabilité de diriger le foyer. Par ailleurs l'analyse de la caractérisation des ménages montre que la majorité des chefs de ménages n'ont aucun niveau d'instruction. Cette situation est beaucoup plus remarquée chez les femmes chefs de ménages. L'analyse du niveau d'instruction des ménages enquêtés fait ressortir 23 chefs de ménages 
polygames instruits (soit 12,10\%) et 30 chefs de menages monogames instruits (soit 15, 15\%). Quant aux chefs de menages en situation de veuvage et de célibat, ils ne représentent que respectivement que $10,81 \%$ et $62,5 \%$ (soit 4 et 5 chefs de ménages instruits) (Tableau 2).

Tableau 2 : Typologie des menages

\begin{tabular}{lllll}
\hline Ménages & $\begin{array}{l}\text { Nombre de } \\
\text { ménages }\end{array}$ & Pourcentage & $\begin{array}{l}\text { Nombre } \\
\text { d'instruits }\end{array}$ & Pourcentage \\
\hline Polygames & 190 & 43,67 & 23 & 12,10 \\
Monogames & 198 & 45,72 & 30 & 15,15 \\
Veufs/Veuves & 37 & 8,54 & 4 & 10,81 \\
Célibataires & 8 & 1,84 & 5 & 62,5 \\
\hline Total & 433 & 100 & 62 & 100 \\
\hline
\end{tabular}

Source: notre enquête, Septembre 2014

\section{Caractéristiques Demographiques Liées Aux Ménages Enquêtés}

La situation démographique de notre zone d'étude se caractérise par des ménages de grande taille. Les résultats laissent apparaitre que seuls 14, $78 \%$ des ménages sont de petite taille c'est-à-dire que le nombre moyen ne dépasse guère les 5 personnes en charge. Les $85,21 \%$ sont des ménages de grande taille et sont repartis comme suit: 126 ménages avec 5 à 10 personnes en charge (soit 29,09\%) ; 203 ménages avec entre 10 à 20 personnes en charge (soit 46,88\%); et 40 ménages ont plus de 20 personnes en charge (soit 9,23\%) (Tableau 3). Toutes ces catégories des ménages ont en commun l'héritage comme principal mode d'accès à la terre. Rares sont celles qui achètent le terrain de culture. Dans la commune urbaine de Tibiri, le mode d'accès à la terre a évolué de l'héritage au don, du don au prêt et du prêt à l'achat avec la nucléarisation des familles. Plus de $90 \%$ des ménages enquêtés ont une faible assise foncière et utilisent la main d'œuvre familiale pour les travaux champêtres. Mais les producteurs à grande assise foncière font recours à la main d'œuvre salariale.

Tableau 3 : Caractéristiques demographiques des ménages enquêtés

\begin{tabular}{lll}
\hline Taille des ménages & Nombre de ménages & Pourcentage \\
\hline de 0 à 5 & 64 & 14,78 \\
De 5 à 10 & 126 & 29,09 \\
De 10 à 20 & 203 & 46,88 \\
Plus de 20 & 40 & 9,23 \\
\hline Total & 433 & 100 \\
\hline
\end{tabular}

Source: notre enquête, Septembre 2014

\section{Principales Speculations Produites, Superficies Emblavées et Rendements Agricoles}

D'après nos enquêtes les types de culture sont essentiellement pluvial et de décrue pratiqué dans la vallée du goulbi. Les principales spéculations produites à travers la culture pluviale sont axées sur la céréaculture (mil, 
sorgho, maïs) et quelques cultures de rente (niébé arachide wandzou). Les spéculations de décrue sont essentiellement 1'Allium cepa (oignon), le Brassica oleracea (chou), Djaxatou (salade), le Solanum tuberosum (pomme de terre), l'Ipomoea batatas (patate douce), le Solanum lycopersicum (tomate), d'Abelmoschus esculentus (gombo), le Lactuca sativa (laitue), le Daucus carota (carotte).

A l'échelle de la zone d'étude, les terres agricoles se raréfient et se dégradent du fait de la pression démographique et du rythme accéléré de la croissance urbaine de ces dernières années.

La tenure foncière est limitée et les superficies emblavées ne permettent pas de produire en quantité suffisante.

En effet, 144 producteurs (soit 33\% de ménages) ne disposent que d'un seul champ ; 196 producteurs (soit $45 \%$ de ménages) possèdent deux champs ; 87 producteurs (soit $20 \%$ de ménages) ont 5 champs et seulement 6 producteurs (soit $2 \%$ de ménages) disposent d'une grande assise foncière (Tableau 4).

Tableau 4: Tenure foncière des ménages

\begin{tabular}{lll}
\hline Tenure foncière & Nombre des menages & Pourcentage \% \\
\hline Un (1) champ & 144 & $33 \%$ \\
Deux (2) champs & 196 & $45 \%$ \\
Cinq (5) champs & 87 & $20 \%$ \\
Plus de cinq champs & 6 & $2 \%$ \\
\hline Total & 433 & 100 \\
\hline
\end{tabular}

Source: notre enquête, Septembre 2014

Il faut noter que les superficies emblavées dans cette zone ont une moyenne de 0,75ha. Ce qui n'est pas sans impact sur le rendement agricole.

Les résultats relatifs aux rendements (Figure 2) montrent que la production varie entre 10 à environ 60 bottes de céréales toutes spéculations confondues. En effet, 30 chefs de ménages récoltent moins de 10 bottes par récolte. Mais, 110 chefs de ménages soit $25 \%$ des enquêtés récoltent moins de 20 bottes ; 143 chefs soit $33 \%$ récoltent entre 20 à 40 bottes ; 102 chefs soit $23 \%$ récoltent environ 40 à 60 bottes et seulement 48 chefs soit $11 \%$ récoltent plus de 60 bottes. Il faut remarquer qu'à ce niveau que plus de la moitié des ménages enquêtés ont une production déficitaire avec $76 \%$ qui récoltent moins de 60 bottes. 


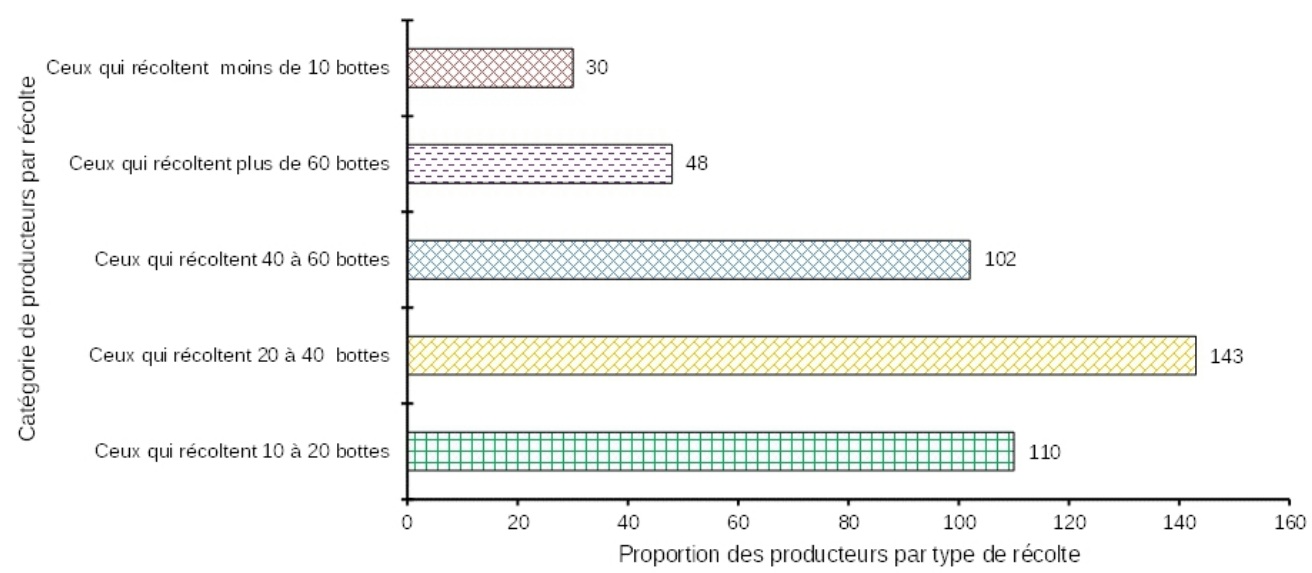

Figure 2: Rendements céréaliers des ménages

De cette faible production, découle le faible revenu des exploitants. Les résultats de notre enquête socio-économique (Tableau 5) ont révélé que ceux qui gagnent moins forment l'écrasante majorité. En effet, $29 \%$ des ménages gagnent moins de $50.000 \mathrm{~F}$ par récolte et $35 \%$ gagnent de 50 à $100.000 \mathrm{~F}$. Ensuite $17 \%$ gagnent de 100 à $200.000 \mathrm{~F}$ et $8 \%$ arrivent à gagner de 200 à $300.000 \mathrm{~F}$. Au contraire, ceux qui produisent mieux ne représentent que $11 \%$ dont $6 \%$ gagnent plus de $400.000 \mathrm{~F}$ et $5 \%$ gagnent de $300.000 \mathrm{~F}$ à $400.000 \mathrm{~F}$ de revenus tirés des produits vendus. D'ores et déjà la production et le revenu de vente des produits sont décroissants dans cette zone.

Tableau 5: Revenus de culture

\begin{tabular}{lll}
\hline Revenus des menages & Nombre de ménages & Pourcentage \\
\hline Moins de $\mathbf{5 0}$ milles & 124 & 29 \\
De $\mathbf{5 0}$ à $\mathbf{1 0 0}$ milles & 152 & 35 \\
De $\mathbf{1 0 0}$ à $\mathbf{2 0 0}$ milles & 74 & 17 \\
De 200 à $\mathbf{3 0 0}$ milles & 34 & 8 \\
De $\mathbf{3 0 0}$ à $\mathbf{4 0 0}$ milles & 23 & 5 \\
Plus de $\mathbf{4 0 0}$ milles & 26 & 6 \\
\hline Total & 433 & 100 \\
\hline
\end{tabular}

Source: notre enquête, Septembre 2014

\section{Couverture Alimentaire des Ménages}

Cette partie nous fait un état de lieu sur la couverture alimentaire des ménages enquêtés (Figure 3). En effet, avec une couverture alimentaire de moins de trois (3) mois ; 48\% des ménages sont dans une situation d'insécurité alimentaire sévère ; $43 \%$ de ménages sont couverts pendant trois (3) à six (6) mois et sont par conséquent touchés par une insécurité alimentaire modérée. Enfin, 7\% des ménages sont à risque et seulement, $2 \%$ ont une couverture de 12 mois sur 12. Il faut noter que la couverture est définie sur la base de leur propre production agricole. De ce fait, si l'insécurité alimentaire se définit 
comme la situation dans laquelle les personnes n’ont pas accès à des quantités suffisantes d'aliments sains et nutritifs pour une croissance normale et pour mener une vie saine et active ; on peut logiquement tirer la conclusion selon laquelle les producteurs de la zone concernée par cette étude sont dans un état de vulnérabilité accrue. Notons que $91 \%$ de ménages sont exposés au danger de cette situation parce qu'ils n'arrivent même pas à couvrir leurs besoins alimentaires pendant la moitié de l'année.

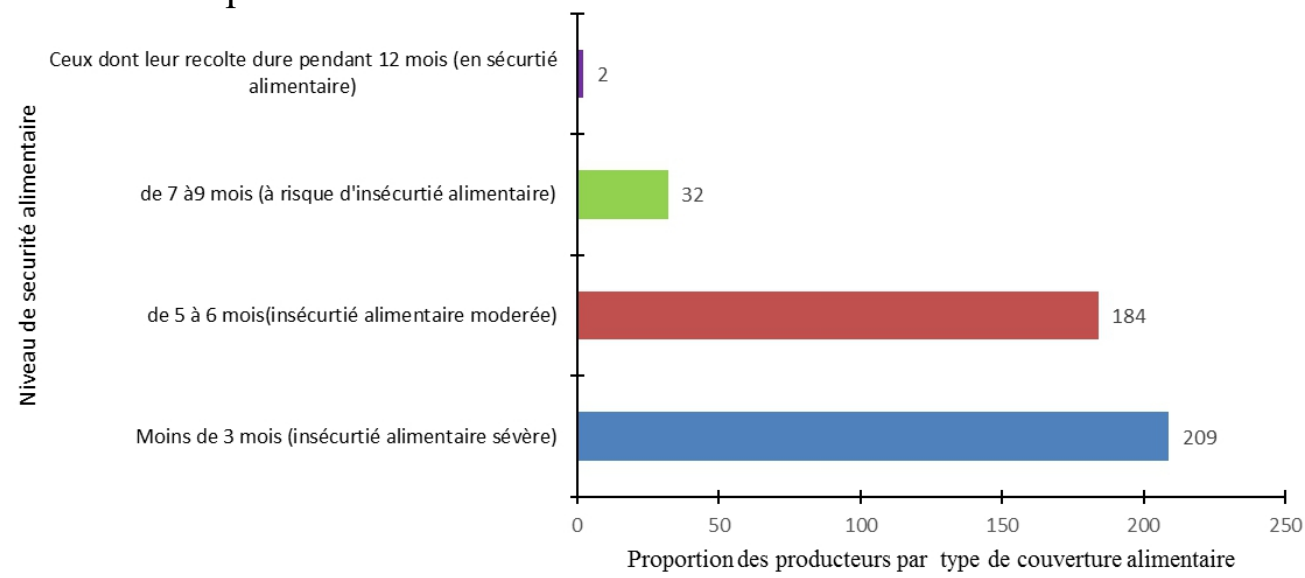

Figure 3: Couverture des besoins alimentaires en mois

\section{Stratégies de Résiliences Face à la Variabilité Climatique Propres à la Zone d'étude}

A l'échelle de la commune de Tibiri, plusieurs stratégies de résiliences apparaissent dont les plus importantes sont celles liées au savoir empirique et local des paysans.

\section{Stratégies d'urgence Selon le Niveau de Vulnérabilité}

Les stratégies d'adaptation face aux chocs climatiques sont multiples et variées. En cas de sècheresse, les ménages les plus vulnérables font recours à des stratégies d'urgence pour subsister avant de mettre en place le processus technique répondant aux modifications de leur environnement. Il s'agit chez les femmes du recours à la mendicité ; aux petits commerces (tels que la vente de tourteau, du henné, de bois et du Moringa). Selon les résultats d'enquête (Tableau 6), 25 chefs de ménages adoptent cette stratégie d'urgence soit 5,77\% de l'effectif de notre échantillon. Ce sont le plus souvent des ménages dirigés par les femmes veuves. Les hommes quant à eux font plutôt recours à l'exode rural, à l'artisanat (fabrication des briques) et au salariat agricole : 207 chefs de ménages $(47,80 \%)$ résistent à travers ces stratégies d'urgence. Ce sont des paysans sans terre et ceux dont la tenure foncière est limitée. Concernant les ménages les moins touchés par les chocs, ils procèdent à la gestion de la main d'œuvre familiale ; c'est-à-dire deux à trois membres de la famille partent en 
exode et les autres restent à la maison pour les travaux champêtres, ceux qui sont en exode envoient de l'argent pour l'achat de la nourriture. Cette catégorie occupe 136 chefs de ménages soit 31\%. C'est la catégorie des ménages de grande taille. Enfin, ceux qui sont à risque d'insécurité alimentaire sont la classe moyenne de la société. Ils conservent leurs aliments jusqu'à l'arrivée de la période de soudure comme ils font des commerces qui leur permettent de s'acheter les vivres dans les marchés. Ils représentent 9,69\% soit 42 chefs de ménages.

Tableau 6: Stratégies d'urgence

\begin{tabular}{lll}
\hline Stratégies d'urgence des ménages & Nombre de ménages & Pourcentage \\
\hline Mendicité et petits commerces & 25 & 5,77 \\
Exode rural, artisanat, salariat agricole & 207 & 47,80 \\
Gestion de la main d'oeuvre familiale & 147 & 33,94 \\
Conservation du stock & 42 & 9,69 \\
En sécurité durant toute l'année & 12 & 2,77 \\
\hline Total & 433 & 100 \\
\hline
\end{tabular}

Source: notre enquête, Septembre 2014

\section{Adaptation Variétale}

Les spéculations les plus cultivées dans la zone sont mises en test variétal par le projet PASADEM (Projet d' Appui à la Sécurité Alimentaire et au Développement de la région de Maradi). Parmi ces spéculations s'observent le mil, le sorgho, le sésame, le niébé et l'arachide. Après avoir testé ces variétés, ce projet s'occupe de leur sélection et de leur vulgarisation dans toute la commune. Elles conviennent à la moyenne des précipitations enregistrées.

Ces variétés sont tolérantes en eau et correspondent au gradient pluviométrique propre à la zone sahélienne. Ce test est conduit par le projet PASADEM qui intervient dans le cadre d'appui à la sécurité alimentaire et au développement de la zone. Il a été mis en pratique expérimentale à travers le champ école paysan, suivi et contrôlé par les techniciens de services agricoles. Ce projet a permis une large diffusion de ces variétés améliorées et hâtives (Tableau 7).

Tableau 7: Test variétal des spéculations

\begin{tabular}{ll}
\hline Spéculations & Variétés sélectionnées \\
\hline Mil & CT6, Zatib, HKP, SOZAT \\
Sorgho & IRAT, Sepon 90 Mota de Maradi, 905 N7, \\
Arachide & JL24, 55-437, RRB, T169-83 \\
Niébé & IT90 ; IT99; KVX ; IT98 ; IT89; TN5-78 \\
\hline Sésame & SN1 ; SN2 ; SN3 ; SN4 ; SN5 ; SN6 ; SN7 ; SN8. \\
\hline
\end{tabular}

Source: Service communal de l'Agriculture de Tibiri (Octobre 2014) 


\section{Ajustement du Calendrier Agricole}

Cette technique consiste à faire des trous du semis dans le bas fond soit deux semaines ou un mois avant la tombée des premières pluies. Les semis se fait lorsqu'on laisse ces trous vides sans rien mettre jusqu'à ce qu'ils reçoivent les deux ou trois premières pluies. Donc c'est à ce moment qu'on va semer le sorgho ou le maïs parce que le trou est déjà imbibé d'eau même en cas d'espacement d'une pluie à une autre, la plante va résister et boucler son cycle végétatif. Mais les trous restent toujours humides et la plante va connaitre un développement rapide qui va aboutir à un rendement satisfaisant. Cette technique se veut une stratégie d'adaptation pour éviter les effets d'arrêts précoces des pluies.

\section{Modification des Pratiques Culturales Comme Stratégies d'adaptation à la Variabilité Climatique}

Les techniques culturales de rétention d'eau de pluie sont appliquées par les paysans dans le bas fond afin d'empêcher le ruissellement quelle qu'en soit la pente du terrain. Elles consistent à faire des sillons croisés sous forme rectangulaire disposés les uns après les autres. Elles rendent et maintiennent la parcelle humide même en cas d'un long espacement de pluie, et enfin permet à la plante de se développer.

La technique culturale d'humidification du sol est aussi appliquée sur les sols ferrugineux tropicaux « Jigawa » sur les champs des plateaux dunaires et hydromorphes des bas fond. Elle consiste à retourner le sol du champ avec la charrue. Dans le bas fond, les paysans renversent d'abord le sol (remaniement précoce) avant de faire les repiquages et les semis. L'importance de cette technique est qu'elle permet au sol de retenir et de conserver l'humidité. Elle permet aussi une bonne pénétration et un bon développement racinaire des plantes. Ce qui aboutira à la production des gros bulbes et des gros épis. Dès qu'on renverse le sol, sa dureté et sa lourdeur diminueront ; donc cet état de légèreté du sol qui permet le développement rapide et florissant de toutes les spéculations de culture.

\section{Adaptation du Système de Culture}

Les paysans mettent en place une technique traditionnelle qui consiste à faire plusieurs semis tout autour des arbustes qui ont un pouvoir fertilisant sur le sol. Ces différentes plantes autour desquelles se passe cette technique permettent un développement rapide du mil et du sorgho. Les spéculations poussent des gros épis et des grosses tiges. Cette stratégie est porteuse par le simple fait que l'on produit beaucoup plus des bottes à travers ces endroits spécifiques que dans le champ en général. En effet, ces arbustes produisent la fumure organique qui fertilise à tout moment le sol. On a entre autres : les semis autour d'Hyphaene thebeica, du Ziziphus moritiana; du Piliostigma 
reticulatum; d'Anona senegalensis; d'Acacia albida du Guiera senegalensis et autour des termitières.

Cette pratique est issue d'un savoir empirique des paysans. Elle est d'une portée avantageuse parce qu'elle permet de booster le rendement agricole et surtout d'accroitre les ressources fourragères.

En outre, ils adoptent une technique au niveau du défrichement des tiges de céréale cultivé: à ce niveau, les paysans ne défrichent pas la partie racinaire du mil/sorgho. Elle est laissée jusqu'à l'arrivée de la saison de pluie et c'est à ce moment qu'on l'a fait ressortir parce qu'elle va servir de fumure pour la fertilisation du sol.

\section{Adaptation Sociale}

L'adaptation sociale n'est autre que le regroupement des exploitants en association pour une synergie d'action pour mieux conduire les activités agricoles. Chaque association a son compte mais la cotisation dépend d'un groupe à un autre. Il y a celles qui cotisent $1000 \mathrm{~F} /$ mois et par membre ; tandis que d'autres cotisent $500 \mathrm{~F}$ et même $250 \mathrm{~F}$ le mois par membre. L'importance de cette cotisation est juste pour alimenter la caisse afin de rendre efficace et durable les associations. L'importance de ces associations est de:

- guider les débutants dans la culture de contre saison;

- partager l'information quand un projet va intervenir dans la commune ;

- donner de crédit d'assistance en cas d'évènement heureux ou malheureux survenu chez un membre de l'association. Mais cette somme sera remboursée après la récolte soit en espèce, soit en nature ;

- de bénéficier des formations sur les techniques culturales modernes et surtout avoir accès aux différents dons des matériels et intrants.

\section{Discussion}

\section{Caractéristiques Sociodémographiques des Ménages}

L'une des caractéristiques de la commune de Tibiri est la croissance accélérée de sa population. Le taux d'accroissement naturel très élevé $(3,7 \%)$ et proche de de la moyenne nationale (3,9\%) (INS, 2014). L'indice synthétique de fécondité est de huit (8) enfants par femme. Cette situation se traduit par une pression accrue sur l'écosystème et accentue la vulnérabilité à l'insécurité alimentaire des ménages (Yamba, 2000 ; Moussa, Yamba, \& Lebailly, 2016).

Les ménages qui parviennent à des rendements déficitaires sont ceux dirigés par les femmes veuves et ceux dont la tenure foncière est très limitée (accès difficile, superficie limitée). Les premiers possèdent des terres laissées par leurs maris mais ne disposent pas des moyens matériels et financiers pour leur mise en valeur efficace. Les deuxièmes sont parfois des paysans sans terre et/ou des paysans ayant un accès difficile et limité aux terres de culture. Ils 
utilisent la main d'œuvre familiale pour le salariat agricole qui constitue leur stratégie de survie (Lawali, Mormont, \& Yamba, 2014).

Toujours dans cette catégorie des paysans sans terres on rencontre des ménages qui ne comptent que sur le système locatif pour se maintenir dans leur milieu tout en vendant leur force de travail ce qui accentue le creusement des inégalités et l'exclusion socio foncière entre les catégories sociales (Lawali et al., 2014). Tous les deux types de ménages sont frappés par le phénomène de la pauvreté et de son cortège malheureux. A ceux-là s'ajoutent aussi les ménages de grande taille qui se baignent sur un seul champ de culture et dont la superficie ne dépasse guère un (1) ha. Bref, la décroissance de la production agricole s'explique par plusieurs paramètres dont entre autres les facteurs socio-économiques vulnérabilisant, les conditions pédoclimatiques hostiles et le système d'exploitation très incontrôlé.

Les trois cas cités sont fortement dépendants de la culture sous pluie. Avec une telle situation qui a un mode d'expression structurelle très remarqué, la vulnérabilité des ménages devient accrue et chronique quand elle s'accouple avec les facteurs conjoncturels propres à l'espace sahélien.

Dans la commune de Tibiri le foncier est un outil précieux et très convoité mais dont la disponibilité est rare. Il subit de plein fouet la pression humaine. Ce phénomène a fait disparaitre la jachère depuis plusieurs années d'où le lessivage progressif des terres agricoles.

\section{Adaptations à la Variabilité Climatique}

Face à la variabilité climatique et dans ses manifestations, les paysans adoptent plusieurs stratégies d'adaptations pour rebondir aux chocs. Mais Selon Agossou, Tossou, Vissoh, et Agbossou (2012), les stratégies paysannes sont fonction des objectifs. Certains paysans cherchent la stabilisation de leurs revenus plutôt que leur maximisation. D'autres cherchent à booster leur production. Dans le cadre de la présente étude, le savoir local est mis en application répondant de prime abord au retard de la tombée des premières pluies, ce qui pousse les exploitants à procéder au labour précoce, à la révision du système de semis, au choix des variétés. Toutes ces stratégies répondent à l'arrivée tardive des précipitations. Elles sont porteuses parce qu'elles permettent à la plante de boucler son cycle végétatif normal et surtout de parvenir à des rendements qui réduisent la vulnérabilité des ménages face aux chocs. Cette analyse corrobore celle de (Diallo, Nacro, \& N'diaye, 2017), qui assertent que les difficultés du secteur agricole causées par le changement climatique et l'activité anthropique ne sont pas restées sans réponse dans le monde. Elles ont connu des mesures d'atténuation à grande échelle auxquelles viennent s'adjoindre des stratégies d'adaptation. Tout de même, les paysans agissent sur le système agraire à travers la modification des techniques culturales et surtout du calendrier agricole. Ces nouvelles techniques 
permettent aux terres de culture de maintenir leur humidité même en cas d'une année pluviométrique déficitaire. Les fruits de ces innovations culturales des paysans se matérialisent à travers la production des gros épis pour les céréales et des gros bulbes pour les tubercules.

Outre ces différentes pratiques adaptatives des paysans, vient s'ajouter l'organisation sociale des exploitants. Le regroupement des exploitants en association est une nouvelle forme d'adaptation qui aboutit à une synergie d'action et d'entre-aide entre les paysans.

Bref, la mise en place des stratégies de résilience s'avère nécessaire pour un rebond efficace aux chocs. Ces stratégies vont contribuer à renforcer les résiliences des populations menacées grâce au développement de leurs capacités, à la fois au niveau institutionnel et communautaire (Issoufou, Boubacar, Adam, \& Yamba, 2017). Cependant, ces stratégies d'adaptation des paysans sont le plus souvent utilisées comme indicateurs indirects d'accès alimentaire. Les paysans commencent à modifier leurs pratiques techniques, à adopter des variétés hâtives, voire d'espèces cultivées pour privilégier des cultures plus rustiques. Les pratiques culturales évoluent également aussi bien sur le plan des dates de réalisation que des techniques employées.

\section{Conclusion}

En définitif, l'étude portant sur l'insécurité alimentaire et les stratégies de résilience dans la vallée de Goulbi Maradi aboutit à l'appréhension d'un certain nombre de facteurs qui expliquent le niveau de vulnérabilité des ménages. Ces facteurs sont liés aux phénomènes naturels (changement climatique, dégradation de sol, insuffisance de précipitation...) et humain (pauvreté, croît démographique). Les résultantes à ces facteurs se résument à l'exposition des ménages agricoles à l'insécurité alimentaire sévère et modérée.

Il est nécessaire de sortir de ce joug d'insécurité et de vulnérabilité alimentaire qui expose la communauté paysanne de la zone d'étude à des risques nutritionnels et sanitaires. Les perspectives pour une bonne résilience des ménages face aux chocs d'ordre naturel et anthropique sont possibles et doivent se traduire par la mise en valeur de la vallée du Goulbi à un niveau si élevé ; ainsi qu'à l'exploitation rationnelle de toutes les ressources à travers un meilleur ciblage d'intervention et des politiques agricoles durables et compatibles avec le secteur.

\section{References:}

1. Agossou, D. S. M., Tossou, C. R., Vissoh, V. P., \& Agbossou, K. E. (2012). Perception des perturbations climatiques, savoirs locaux et stratégies d'adaptation des producteurs agricoles béninois. African Crop Science Society 20, 565 - 588. 
2. Boubacar, Y. (2000). Évolution des régimes de propriété et d'utilisation des ressources naturelles dans la région de Maradi. Drylands Research.

3. Deressa, T. T., Hassan, R. M., \& Ringler, C. (2011). Perception of and adaptation to climate change by farmers in the Nile basin of Ethiopia. The Journal of Agricultural Science, 149(1), 23-31. https://doi.org/10.1017/S0021859610000687.

4. Diallo, S., Nacro, H., \& N'diaye, A. (2017). Efficience des stratégies endogènes d'adaptation du secteur agricole aux changements climatiques dans le bassin de la région de Thiès (Sénégal). International Journal of Biological and Chemical Sciences, 11(2): 707721. http://ajol.info/index.php/ijbcs.

5. Eromosele, C. O., Arogundadea, L. A., Eromosele, I. C., \& Ademuyiwa, O. (2008). Extractability of African yam bean (Sphenostylis stenocarpa) protein in acid, salt and alkaline aqueous media. Food Hydrocolloids 22 (2008) 1622-1628. https://www.elsevier.com/locate/foodhyd.

6. Heijmans, A. (2013). Reaching Resilience - Manual Resilience 2.0 pour les acteurs de l'aide et les décideurs Humanitarian Response. WWw.reachingresilience.org.

7. Heinrigs, P. (2007). La zone écologique. Série environnement. Atlas de l'intégration régionale fragile des pays du sahel. https://www.oecd.org/fr/csao/.

8. Issoufou, O., Boubacar, S., Adam, T., \& Yamba, 8. (2017). Au Niger l'adoption des nouvelles stratégies par les producteurs est l'une des voies du développement de l'agriculture pluviale. African Crop Science Journal, 25(2), 207 - 220.

9. Lawali, S., Mormont, M., \& Yamba, B. (2014). Gouvernance et stratégies locales de sécurisation foncière : étude de cas de la commune rurale de Tchadoua au Niger. VertigO.14(1). https://doi.org/10.4000/vertigo.14723.

10. Mortimore, M. J., \& Adams, W. M. (2001). Farmer adaptation, change and 'crisis' in the Sahel. Global Environmental Change, 11(1), 49-57. https://doi.org/10.1016/S0959-3780(00)00044-3.

11. Moussa, K., Yamba, B., \& Lebailly, P. (2016). Insecurité alimentaire et pauvreté des menages en milieu rural au Niger : cas de la region de Tahoua. Algerian Journal of Arid Environment, 6, 4-15.

12. Ouédraogo, D., Kaboré, M., \& Kienou, B. (2007). Insécurité alimentaire, vulnérabilité et pauvreté en milieu rural au Burkina : une approche en termes de consommation d'énergie. Mondes en développement, 4 (140) ,65-84. http://www.cairn.info.DOI $: 10.3917 / \mathrm{med} .140 .0065$. 
13. Rahman, M., Luo, J., Hafeez, A., \& Sun, T. (2015). A Comprehensive Review of Microfinance Impacts, Sustainability and Outreach. Asian Journal of Agricultural Extension, Economics \& Sociology, 6(2), 64-76. https://doi.org/10.9734/AJAEES/2015/16513.

14. Sadda, A.-S., Lawali, S., Diouf, A., Illiassou, A., \& Mahamane, A. (2016). Land Crisis and Its Impacts on Agropastoral Systems in the Commune of Tibiri (Maradi Region) / Niger. Int. J. Pure Appl. Sci. Technol., 33(1) - 37(2) (2016), 1-10.

15. Srivastava, A., Shakya, A. K., \& Kumar, A. (2012). Boronate affinity chromatography of cells and biomacromolecules using cryogel matrices. Enzyme and Microbial Technology, 51(6-7), 373-381. https://doi.org/10.1016/j.enzmictec.2012.08.006.

16. INS (2014). Annuaire statistique du Niger 2010-2014.pp-69.

17. Vodounou, J. B. K., \& Onibon Doubogan, Y. (2016). Agriculture paysanne et stratégies d'adaptation au changement climatique au Nord-Bénin. Cybergeo. https://doi.org/10.4000/cybergeo.27836.

18. Vonthron, S., Dury, S., Fallot, A., Alpha, A., \& Bousquet, F. (2016). L'intégration des concepts de résilience dans le domaine de la sécurité alimentaire: regards croisés. Cahiers Agricultures, 25(6), 64001. https://doi.org/10.1051/cagri/2016039.

19. Yabi, I., \& Afouda, F. (2012). Extreme rainfall years in Benin (West Africa). Quaternary International, 262, 39-43. https://doi.org/10.1016/j.quaint.2010.12.010. 